"Industrial Activities" section. While international trade has played and continues to play a highly significant role in the growth and expansion of South Korea's industrial activities, this topic is discussed only briefly (150-151) and is not emphasized in the subsections detailing individual manufacturing and service industries.

Despite its shortcomings, Volume III of The National Atlas of Korea: Human Geography is an important contribution to furthering our geographical knowledge and awareness of current issues in South Korea. It is an authoritative source of facts, statistics, and of course maps about South Korea's people, economy, culture, cities, industries, politics, and other human geographical features.

\section{OBTAINING THIS ATLAS}

All volumes of The National Atlas of Korea are available online for viewing or download at no cost by visiting nationalatlas.ngii.go.kr.

Distribution of the five hardcover volumes of the atlas has been delegated to the Korean Geographical Society (KGS). We are told that KGS is distributing a very limited number of the books for $\$ 150$ per volume (excluding shipping). It is suggested that interested parties contact KGS directly at this address: Korean Geographical Society. 1413-ho, 213-12. Saechang-ro. Yongsan-gu. Seoul. 140871. Korea.

\title{
GEODESY: INTRODUCTION TO GEODETIC DATUM AND GEODETIC SYSTEMS
}

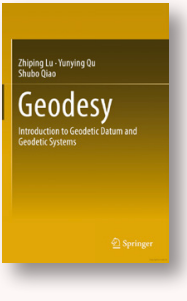

Zhiping Lu, Yunying Qu, and Shubo Qiao

Springer, 2014

401 pages, \$129.99 softcover, \$99 eBook

ISBN: 978-3-642-41244-8

Review by: Fritz Kessler, The Pennsylvania State University

Where is $13^{\circ} 42^{\prime} 56^{\prime \prime}$ and $127^{\circ} 47^{\prime} 33^{\prime \prime}$ located? This seemingly simple question is fraught with complexities. Let's begin by assuming that the latitude value is first and longitude is listed second. Next, one would need to know the cardinal direction (north or south latitude and east or west longitude) associated with each value. If we say that $13^{\circ} 42^{\prime} 56^{\prime \prime}$ is south and $127^{\circ} 47^{\prime} 33^{\prime \prime}$ is east, then this point is located in or near East Grace Park, Caloocan, in The Philippines. However, to precisely and accurately tie these coordinates to a location on the Earth's surface requires a datum. A datum provides the horizontal and vertical control that allows determination of accurate coordinate locations and elevations. Datums are important concepts in the field of geodesy, and are just one of the range of classical and modern themes in that field that are the subject of Geodesy: Introduction to Geodetic Datum and Geodetic Systems. This textbook-the authors of which are on the faculty of People's Liberation Army Information Engineering University in Zhengzhou, Henan Province, People's Republic of China-is an English edition of a text that was originally published in Chinese in 2006.
Geodesy begins with a preface that explains the motivation for pursuing this topic. It is followed by an abbreviations reference section that defines the dozens of acronymsacronyms that can be challenging even to an experienced reader-that appear throughout the book. The table of contents provides a very detailed description of each chapter's contents, and there is also a one-page biographical overview of the authors and their affiliations.

Chapter 1 begins with an introduction to geodesy and its objectives, and then surveys the fields that fall under that rubric. Applications of geodesy in fields such as engineering construction, geosciences, and disaster prevention are discussed in turn, followed by the historical development of different forms of geodesy-physical, satellite, and dynamic. Other topics briefly discussed in the latter sections of the chapter include space geodesy, satellite navigation, and the Earth's gravity field. A short Review and Study Questions section closes this chapter, and is a feature found in all chapters in this book.

Chapter 2, "Geodetic Data Collection Techniques," details the specific measurements, techniques, and instrumentation that can be used to derive data for geodetic purposes. It is divided into two parts, the first of which provides an overview of various measurements that are characteristically collected for geodetic purposes, including horizontal and vertical angles, distances, and astronomic observations such as astronomic latitude, longitude, and azimuth. This part also discusses the equipment used for that collection, and the collection methods employed. Tools of the trade, 
such as the theodolite and electromagnetic distance measuring devices, are described, and methods such as leveling are explained. The second part of the chapter details the Global Positioning System (GPS), beginning with an overview of GPS and its various components. Other GPSrelated topics discussed include measurement and positioning (pseudo-range, absolute, and real-time), satellite laser ranging (SLR), the very long baseline interferometry (VLBI) system, satellite altimetry (used, for example, to measure the velocity of ocean currents), and gravimetry (useful in determining gravitational acceleration).

Chapter 3, "Geodetic Datum and Geodetic Control Networks," covers horizontal and vertical datums. Several topics related to the creation of a horizontal datum are described, including the methods of establishing a control network (traversing and triangulation), accuracy hierarchies (first-, second-, third-, and fourth-order), density of control points, and reconnaissance for monument site selection. There is an interesting section on how to erect a monument or survey control point for horizontal control. A discussion of vertical datums is next, and begins with the importance of leveling and continues with a treatment of the process of establishing a vertical datum in China. The process of integrating horizontal and vertical datums into a three-dimensional coordinate datum is tackled in the last section of this chapter. Latitude, longitude, ellipsoid height, and velocities measured by orbiting Earth's satellite can be integrated within a four-dimensional geometric datum, something not possible using classical horizontal and vertical datums because they are normally treated as separate one-dimensional frameworks. GPS, VLBI, and SLR systems such as EUROLAS or Global IVS Network provide the infrastructure needed to define three-dimensional coordinate datums. China's efforts to establish its own three-dimensional coordinate datumthe China Gravity Basic Network 1957, 1985, and 2000are discussed in the concluding part of the chapter.

Chapter 4, "The Geoid and Different Height Systems," presents an overview of height and how it is determined. The chapter starts by reviewing gravity and explaining its role in describing the Earth's shape. Included in this discussion is an overview of the Earth's treatment as an ellipsoid. The crux of the height discussion comes next, with a review of the different characterizations of height: orthometric, normal, dynamic, and geodetic height. Following this discussion is a description of transformation methods between different height systems.
Chapter 5, "Reference Ellipsoid and the Geodetic Coordinate System," focuses on the relationship between geodetic measurements of the Earth's surface and how those measurements can be associated with-or reduced to- - a chosen ellipsoid. Discussion begins with a review of spherical trigonometry that includes topics such as spherical triangles and trigonometric identities. The reference ellipsoid is again revisited, but this time the focus is on a detailed presentation of the various defining parameters and their interrelationships. This information is necessary equipment for the reader's understanding of the rest of the chapter-an examination of the formulaic relationships between different coordinate systems (for example, between a geodetic coordinate system and the geodetic spatial rectangular coordinate system). Some of the topics in this discussion include the understanding and derivation of the radius of curvature in the prime vertical, the radius of curvature in the prime meridian, and the mean radius of curvature, all of which are useful when defining coordinate systems. The next section presents a thorough overview of the geodesic and its properties on the reference ellipsoid, followed by a discussion of reducing geodetic observations made on the Earth's physical surface to a chosen reference ellipsoid-an important step in the creation of accurate coordinates.

Chapter 6, "Gauss and UTM Conformal Projections and the Plane Rectangular Coordinate System," focuses on representing reduced geodetic measurements, defined on a reference ellipsoid, using conformal map projections and plane rectangular coordinate systems. The chapter begins with a concise overview of the role that map projections play in general, and the uniqueness of conformality in particular. The Gauss-Krüger conformal projection and its historical development is the focal point of the discussion, particularly in the context of its role in the Universal Transverse Mercator (UTM) system. This discussion pays particular attention to the numerous Gauss-Krüger formulae that mathematically describe, for example, the projection's distortion, scale factor determination, and the forward and inverse ellipsoidal forms of the projection plotting coordinates. The chapter concludes with a discussion of the characteristics a Gauss plane imposes on such concepts as true and grid north, grid convergence computations, arc-to-chord and distance correction, and scale factor calculations.

Chapter 7, "Establishment of Geodetic Coordinate Systems," reviews the concept of a terrestrial reference 
system and the practices of establishing of such a system. The chapter starts by introducing transformation methods between, for example, different geodetic coordinate systems and different geodetic Cartesian coordinate systems. Attention focuses next on the geocentric coordinate system itself, and its many application. Some of these applications include construction layout, navigation, and space technology. The conventional terrestrial reference system (CTRS) and coordinate terrestrial reference frame (CTRF) are both explained in detail. This preliminary discussion paves the way for a comprehensive look at the different geodetic coordinate systems in use by China. The bulk of the attention is focused on the China Geodetic Coordinate System 2000.

An extensive bibliography of approximately 250 sources and an index concludes the book.

Like many fields, geodesy has witnessed important technological changes in recent decades. Geodesy: Introduction to Geodetic Datum and Geodetic Systems presents a comprehensive overview of the field that includes both classical approaches to geodetic measurement techniques and modern practices with satellite geodesy. There isn't anything shockingly new regarding the treatment of classical geodetic methods here-in fact, other texts provide a more comprehensive review of classical geodesy. However, that is not is not this book's strength, which lies in explaining the connection between classic and modern geodesy. This bridge is clearly present in the authors' Chapter
7 discussion of China's evolution of a geodetic coordinate system, which takes the reader through an interesting journey detailing decades of coordinate system development.

An allied strength of Geodesy comes in the considerable breadth of material presented. While some geodesy texts may delve into great detail on a selected number of topics, Geodesy discusses an extensive list of topics that widely samples from the field. While the discussions are at times thinly presented, the reader is introduced to many topics that might otherwise not be covered. Most topics are described in sections comprised of short paragraphs, making it easy for the reader to quickly grasp the material. Copious equations are found throughout the chapters, many of which include worked examples. To help elucidate the topics, the authors have provided many real-world examples. There are numerous illustrations includedmostly black and white line drawings- that help to illustrate specific concepts. A separate listing of the figures and equations would have made it easier for the reader to find a specific one, but this helpful addition is not included.

Overall, this is not a book for the faint of heart. It is packed full of dense information on a topic that is fundamentally complex. One should think of this book as more encyclopedic in its presentation instead of something that is to be read cover to cover-although certain readers will undoubtedly want to take that approach. If your work involves geodesy, or is tangentially related to the field, this book is an important reference.

\section{THE FENCE AND THE BRIDGE: GEOPOLITICS AND IDENTITY ALONG THE CANADA-US BORDER}

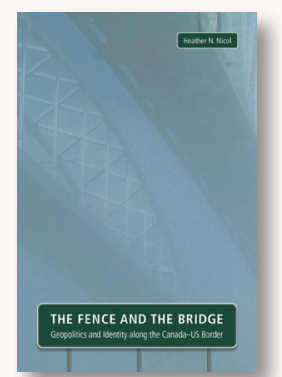

By Heather N. Nicol

Wilfrid Laurier University Press, 2015

296 pages, $\$ 34.39$ softcover, $\$ 39.58$ Kindle e-book.

ISBN: 978-1-55458-971-5

Review by: James C. Saku, Frostburg State University

Humans tend to claim ownership of specific territories, and boundary issues between individuals, groups, and countries are nothing new. They have been common both among nomadic groups and among those living more sedentary lives. It is unsurprising, then, that Canada and the United States have had a long history of border disputes arising from various economic and political issues.

The Fence and the Bridge: Geopolitics and Identity along the Canada-US Border is an engaging book that presents a very comprehensive analysis of the historical and contemporary issues that have shaped the border between these two nations. The first of its eight chapters examines the Canada-United States border relationship in the late eighteenth and early nineteenth centuries. At that time, the border between both countries was considered an unstable frontier that effectively drifted north and south. The author, Heather Nicol, notes that while Canadians portrayed 